\title{
THE HUTCHINSON DIETETIC HYPOTHESIS OF FISH EATING AS A CAUSE OF LEPROSY. A REAPPRAISAL IN THE LIGHT OF THE INFLUENCE OF PRO-OXIDANT NUTRITIONAL CONDITIONS.
}

\author{
by MenY Bergel, M.D., \\ Director of the Leprosy Research Laboratory, \\ Rosario, Argentina.
}

In 1906 Jonathon Hutchinson published a book which summarised his observations on some diets which favoured the development of human leprosy. In many countries he found a suggestively high incidence of leprosy in peoples who ate large amounts of decomposing fish. He said "the cause of the disease is some ingredient or parasite generated by or introduced into the fish which has either been not cured at all, or cured badly: it is however quite possible that the ingredient present in the fish may be something quite different from the bacillus itself and that it may even be of chemical nature".

The so-called "fish hypothesis" of leprosy, which came into being as the result of the conclusions of Hutchinson, became the basis of an intensive search for the Hansen bacillus in decaying fish. When the results of this search proved to be completely negative, the postulates of Hutchinson gradually lost strength and were finally abandoned altogether and are quoted today only for historical reasons, although it will be noted that this was not entirely logical, since Hutchinson had specifically stated that the factor in fish might be something other than the Hansen bacillus.

Recent experimental studies and interpretations by the author make it seem desirable to reconsider the ideas of Hutchinson. It now appears plausible to explain the well-established correlation between the occurrence of leprosy and the ingestion of diets high in decomposing fish on the following grounds. Decomposing fish contain relatively large quantities of rancid fats and unsaturated fatty acids, which would be expected to induce a prooxidant condition in the tissues of persons ingesting such fish, and it can be shown that a prooxidant condition favours the growth of the Hansen bacillus. This is shown as follows:

(a) The Hansen bacillus can be made to multiply in rats fed a prooxidant diet. Three serial intratesticular inoculations of Hansen bacilli were made in rats kept on a pro-oxidant diet (low in Vitamin E and containing 15\% linseed oil), during a period of 26 months. Seven months after the last inoculation the testes were found to contain considerable numbers of acid-fast bacilli ${ }^{2,3,4}$ which were tested biologically in two ways. 
In the first test these bacilli were seeded on Lowenstein-Jensen media and were found not to develop colonies at various temperatures, up to a period of 120 days. In other words, the bacilli would not grow in an artificial medium designed for acid-fast bacilli ${ }^{4}$ and in this respect resemble Hansen bacilli. In the second test, a lepromin made with testicular tissue from inoculated rats was tested in the usual manner ${ }^{5}$ along with integral and bacillary lepromins made from human lepromas, and the rat tissue lepromin was found to behave in the same way as the lepromin from the human tissues.

These two biological tests showed that the bacilli found in the testes of the inoculated rats were Hansen bacilli. Likewise, a comparative study has been made of the growth of $M$. leprae inoculated intratesticularly in white rats submitted to various pro-oxidant nutritional conditions. With the prooxidant diet employed (Vitamin E deficient diet with linseed oil, with rancid linseed oil, with cod liver oil, with or without the addition of silver nitrate in the drinking water and injection of haemolysates) there was a notable growth of $M$. leprae in relation to their growth in control animals fed on ordinary diets ${ }^{6}$.

(b) It was possible to demonstrate an antioxidant activity in vivo of various antileprotic compounds as follows. Rats kept on a pro-oxidant diet (low in Vitamin E and containing 15\% cod liver oil) were found to be protected for periods up to five months against the formation of ceroid pigment in the subcutaneous, perigonadal and perirenal fat by the addition to the diet of $0.2 \%$ isoniazid, $0.2 \%$ diaminodiphenylsulphone, $0.1 \%$ 4-acetylaminobenzaldehyde thiosemicarbazone and $0.5 \%$ 4-butoxy $-4^{1}$ dimethylamonothiocarbanilide. Control animals not given these drugs formed ceroid pigment in the above mentioned tissues in large amount ${ }^{7,8,9}$. These experiments show that these antileprotic drugs have antioxidant activity.

(c) But compounds known to have high antioxidant activity and not previously used against leprosy were found to exhibit antilepromatous activity. For instance, the administration of large amounts of Vitamin E (600-800 mgm. daily) to a group of lepromatous leprosy cases was found to exert a very remarkable antilepromatous effect ${ }^{10}$. From the experimental findings presented above, it is concluded that there is a very clear relationship between the pro-oxidant state, which favours the autoxidation of lipids, and the pathogenesis of leprosy ${ }^{11}$. The pro-oxidant state with the accompanying autoxidation of lipids can be produced by the ingestion of decomposing fish. This thus appears to lend support to the "fish hypothesis" as 
it was originally postulated by Hutchinson, and moreover indicates the nature of the material in the decaying fish which favours the development of leprosy.

\section{References}

1. Hutchinson, J. "On Leprosy and Fish-Eating". (1906) Constable, London.

2. Bergel, M. (1957) Sem Medica, 111, 479.

3. Bergel, M. (1957) Sem. Medica, 111, 1148.

4. Bergel, M. (1957) Sem. Medica, 111,1313.

5. Bergel, M. (1959) I. Journ. Leprosy, 27, 59.

6. Bergel, M. (1959) Leprosy Review, 30, 153.

7. Bergel, M. (1957) Sem. Medica, 110, 192.

8. Bergel, M. (1957), Sem Medica, 110, 855.

9. Bergel, M. (1958) Prensa Med. Argent., 45, 2565.

10. Bergel, M. (1958) Leprologia, 3, 133

11. Bergel, M. (1958) I. Journ. Leprosy, 26, 65. 